\title{
Tuberculosis meníngea: reporte de casos años 2005-2017
}

\author{
Meningeal tuberculosis: cases report years 2005-2017
}

Diego Rodríguez', Alejandra Zapata², Florencia Molina', Griselda Perruolo³, Johnny Burbano³ y Laydi Correa

${ }^{1}$ Medicina Interna, Hospital Nacional Prof. A. Posadas. Buenos Aires, Argentina.

2Servicio de Laboratorio, Sector de Bacteriología, Sección Micobacterias, Hospital Nacional Prof. A. Posadas. Buenos Aires, Argentina.

${ }^{3}$ Residentes del servicio de medicina interna, Hospital Nacional Prof. A. Posadas. Buenos Aires, Argentina.

Conflicto de intereses: Ninguno para declarar.

Financiamiento: Financiamiento propio.

Recibido: 13 de diciembre de 2020 / Aceptado: 30 de marzo de 2021

\section{Resumen}

Introducción: La tuberculosis (TBC) continúa siendo un problema de salud pública mundial; su forma meníngea conlleva mayor letalidad y secuelas, en particular si se asocia a la infección por VIH/SIDA. Objetivo: Describir las características demográficas, presentación clínica, laboratorio y de las imágenes de los pacientes con TBC meníngea (aislamiento de Mycobacterium tuberculosis en LCR), analizando diferencias entre pacientes con y sin infección por VIH/SIDA. $P a$ cientes y Métodos: Estudio observacional y descriptivo, retrospectivo, de una serie de casos atendidos en el Hospital Dr. Alejandro Posadas de Buenos Aires, Argentina, desde enero de 2005 hasta diciembre de 2017. Resultados: Se analizaron 36 pacientes, 22 de ellos mujeres, con una mediana de edad de 36,5 años. Veintidós pacientes presentaron co-infección por VIH, todos en estadio SIDA. El tiempo de inicio de síntomas tuvo una mediana 11 días. predominando fiebre, estado de conciencia alterado y cefalea. En el LCR se hallaron linfocitosis, hipoglucorraquia, hiperproteinorraquia y ácido láctico elevado. Se realizó tomografía computada de encéfalo a 34 pacientes, 16 sin alteraciones. En otros 16 se realizó resonancia magnética (RM) cerebral, 9 presentaban trastornos vasculares. La RM fue más sensible para identificar refuerzo meníngeo, trastornos de tipo vasculares, y lesiones de tipo granulomatosas. La mediana de inicio de tratamiento fue de 1 día, con $72,2 \%$ recibiendo co-adyuvancia con corticosteroides. La mortalidad observada fue de $27,7 \%$ y secuelas hubo en $36,1 \%$. Sólo 5 pacientes requirieron intervención neuro-quirúrgica. Conclusión: Siendo la TBC meníngea una afección de alta morbimortalidad, es imperioso asegurar un diagnóstico temprano en su evolución mediante la incorporación de la biología molecular e imagenología (RM) al amplio uso clínico.

Palabras clave: tuberculosis; meningitis; virus de inmunodeficiencia humana; síndrome de inmunodeficiencia adquirida.

\section{Abstract}

Background. Tuberculosis (TB) continues to be a global public health problem; its meningeal form leads to greater lethality and sequelae, particularly if it is associated with HIV / AIDS infection. Aim: To describe the demographic characteristics, clinical presentation, laboratory and images of patients with meningeal TB (isolation of Mycobacterium tuberculosis in CSF), analyzing differences between HIV and non-HIV patients. Methods: We performed an observational and descriptive study, with retrospective analysis of patients attending at the Dr. Alejandro Posadas Hospital, Buenos Aires, since January 2005 to December 2017. Results: Thirty-six patients were analyzed, with 22 women with a median age of 36.5 years. Twenty two patients had HIV coinfection, all in the AIDS stage. The symptom onset time was median 11 days. The predominant ones were fever, altered consciousness and headache. In the cerebrospinal fluid were lymphocitosis, hypoglycorrhachia, hyperproteinorrhachia and high lactic acid, according to previously described findings. Of 34 patients who underwent brain scan, 16 patients had no significant pathological findings. MRI was performed in 16 patients, 9 had vascular disorders. Brain MRI was more sensitive to identify meningeal reinforcement than computerized tomography, vascular disorders, and granulomatous lesions. The median onset of treatment was 1 day, with $72.2 \%$ of the total receiving coadjuvants with corticosteroids. Mortality of $27.7 \%$ and sequelae in $36.1 \%$ were observed. Only 5 patients required neurosurgical intervention. Conclusion: Since meningeal TB is a disease with high morbidity and mortality, it is imperative to ensure an early diagnosis in its evolution by incorporating molecular biology and imaging (MRI) into broad clinical use.

Keywords: tuberculosis; meningitis; human immunodeficiency virus; acquired immunodeficiency syndrome. 
las características demográficas, hallazgos clínicos, de laboratorio y de las imágenes, en los pacientes con diagnóstico definitivo confirmado mediante el aislamiento de $M$. tuberculosis en el LCR, desde enero de 2005 hasta diciembre de 2017 en nuestra institución y comparar los resultados hallados con los descritos en la literatura. Como objetivos secundarios nos proponemos: describir el tratamiento administrado, analizar la mortalidad de los pacientes durante la internación; la presencia de secuelas; el requerimiento o no de métodos neuro-quirúrgicos y comparar los resultados encontrados entre pacientes con infección por VIH y sin ella, con lo descrito en la literatura médica

La bibliografía analizada cuenta con un porcentaje verdaderamente pequeño de casos realmente confirmados de este cuadro por aislamiento bacteriológico, por lo que consideramos de relevancia analizar la casuística regional en los casos confirmados.

\section{Pacientes y Métodos}

Se realizó un estudio observacional y descriptivo, con análisis retrospectivo, a partir de la revisión de historias clínicas en el archivo del hospital según la codificación del diagnóstico de egreso del CIE-10, compatible con TBC meníngea o meningitis tuberculosa, además de la búsqueda en los libros de registro de bacteriología de nuestro hospital, en la sección de micobacterias, para estudiar todos los casos con aislamiento de M. tuberculosis en LCR, y analizar además la sensibilidad in vitro de dichos aislados.

\section{Criterios de inclusión}

Pacientes mayores de 18 años y con diagnóstico definitivo de TBC meníngea por aislamiento de $M$. tuberculosis en LCR.

\section{Criterios de exclusión}

Pacientes bajo 18 años de edad.

Las variables analizadas de las historias clínicas fueron: características demográficas (sexo y edad), enfermedades subyacentes (co-infección con VIH), presentación clínica (tiempo de inicio de los síntomas expresado en días, signos y síntomas al ingreso, estadio de la enfermedad según Medical Research Council (Estadio I: Síntomas inespecíficos, sin alteración de la conciencia ni signos neurológicos. Estadio II: Cierto grado de confusión mental y aparición de signos neurológicos leves. Estadio III: Signos de foco motor, compromiso de la conciencia, convulsiones), los datos de laboratorio (análisis del LCR: glucosa, proteínas, ácido láctico, recuento celular), hallazgos bacteriológicos (aislamiento de M. tuberculosis), estudios de imágenes 
(realización de RM y/o TC de cerebro y sus hallazgos), uso de corticoesteroides (dexametasona) y tratamiento antituberculoso elegido (isoniazida en dosis de $5 \mathrm{mg} / \mathrm{kg}$ / día, rifampicina en dosis de $10 \mathrm{mg} / \mathrm{kg} /$ día, etambutol en dosis de $20 \mathrm{mg} / \mathrm{kg} /$ día o estreptomicina en dosis de 20 $\mathrm{mg} / \mathrm{kg}$ intramuscular y pirazinamida en dosis $25 \mathrm{mg} / \mathrm{kg} /$ día), intervenciones quirúrgicas realizadas (derivación ventricular externa, derivación ventrículo-peritoneal), mortalidad durante la internación y condiciones clínicas al alta (presencia de secuelas neurológicas).

\section{Análisis del LCR}

Obtenido el LCR se le efectuó análisis físico-químico, examen directo (tinción de Ziehl-Neelsen) y cultivo en

Tabla 1. Características demográficas y clínicas de los 36 pacientes con diagnóstico definitivo de tuberculosis meníngea

$\begin{array}{lll}\text { Sexo (Total, porcentaje) } & & \\ \text { Mujeres } & 22 & 61,1 \% \\ \text { Hombres } & 14 & 38,9 \%\end{array}$

$\begin{array}{ll}\text { Edad (años) } & \text { Mediana (mínimo y máximo) } \\ \text { Todos } & 36,5(18-85) \\ \text { Mujeres } & 36(18-85) \\ \text { Hombres } & 36,5(21-65)\end{array}$

Duración de los síntomas en días (mediana, rango)

Todos

$11(1-120)$

\begin{tabular}{|c|c|c|c|}
\hline \multicolumn{4}{|c|}{ Infección VIH (Porcentaje del total de pacientes de mismo sexo) } \\
\hline Todos & 22 & $61,1 \%$ & \\
\hline Mujeres & 13 & $59,1 \%$ & \\
\hline Hombres & 9 & $64,3 \%$ & \\
\hline Clínica al momento de la consulta & Total (\%) & $\begin{array}{l}\text { VIH (\%) } \\
n=22\end{array}$ & $\begin{array}{c}\text { No VIH (\%) } \\
n=14\end{array}$ \\
\hline Fiebre & $28(77,7 \%)$ & $16(72,7 \%)$ & $12(85,7 \%)$ \\
\hline Estado de conciencia alterado & $26(72,2 \%)$ & $15(68,2 \%)$ & $11(78,6 \%)$ \\
\hline Cefalea & $21(58,3 \%)$ & $10(45,4 \%)$ & $11(78,6 \%)$ \\
\hline Rigidez de nuca & $19(54,5 \%)$ & $10(45,4 \%)$ & $9(64,3 \%)$ \\
\hline Otros & $31(86,1 \%)$ & $20(90,9 \%)$ & $11(78,6 \%)$ \\
\hline $\begin{array}{l}\text { Clasificación según Medical } \\
\text { Research Council al ingreso }\end{array}$ & Total (\%) & $\begin{array}{l}\text { VIH (\%) } \\
n=22\end{array}$ & $\begin{array}{c}\text { No VIH (\%) } \\
n=14\end{array}$ \\
\hline Estadio I & $7(19,5 \%)$ & $5(22,8 \%)$ & $2(14,3 \%)$ \\
\hline Estadio II & $19(52,7 \%)$ & $10(45,4 \%)$ & $9(64,3 \%)$ \\
\hline Estadio III & $10(27,8 \%)$ & $7(31,8 \%)$ & $3(21,4 \%)$ \\
\hline Tuberculosis en otras localizaciones & Total (\%) & $\begin{array}{l}\text { VIH (\%) } \\
n=22\end{array}$ & $\begin{array}{c}\text { No VIH (\%) } \\
n=14\end{array}$ \\
\hline No & $19(52,7 \%)$ & $10(45,4 \%)$ & $9(64,3 \%)$ \\
\hline Pulmonar & $6(16,7 \%)$ & $5(22,8 \%)$ & $1(7,1 \%)$ \\
\hline Diseminada & $10(27,8 \%)$ & $7(31,8 \%)$ & $3(1,5 \%)$ \\
\hline Otras localizaciones & $1(2,8 \%)$ & 0 & $1(7,1 \%)$ \\
\hline
\end{tabular}

medio sólido (Lowenstein-Jensen) y medio líquido (método fluorométrico BACTEC ${ }^{\circledR}$ MGIT 960 BD). A partir de los cultivos positivos se realizó la identificación del complejo M. tuberculosis por NAP-test (años 2005 a 2010) y posteriormente por el método de inmunocromatografía lateral (TBid) (BD). Las pruebas de sensibilidad in vitro se realizaron por el método fluorométrico (MGIT). En las fechas que comprende el estudio, la institución aún no contaba con la determinación de reacción de polimerasa en cadena (RPC) en LCR, técnica que fuera incorporado al laboratorio a partir del año 2018.

La información fue expresada en forma estandarizada en una planilla de Microsoft Excel ${ }^{\circledR}$, para su análisis.

\section{Análisis estadístico}

Las variables numéricas se resumieron como medidas de posición central, ya fueran medias o medianas según su distribución y las variables categóricas se expresaron como porcentaje del total correspondiente.

\section{Resultados}

Se llegó a un total de 36 pacientes con aislamiento de M. tuberculosis en cultivo de LCR; las características demográficas, clínicas y hallazgos de TBC en otras localizaciones de los pacientes analizados se presentan en la Tabla 1. Todos los aislados fueron sensibles a estreptomicina, isoniacida, rifampicina, y etambutol.

De los 36 pacientes, 22 eran mujeres $(61,1 \%)$, y la mediana de edad era de 36,5 años, con un rango entre $\operatorname{los} 18$ y 85 años.

Del total de pacientes, 22 presentaban co-infección por VIH al momento del diagnóstico $(61,1 \%)$, siendo 13 mujeres $(59,1 \%$ del total de mujeres) y 9 hombres $(64,3 \%$ del total de hombres), todos ellos en etapas avanzadas de la enfermedad (SIDA), con irregular o nulo tratamiento anti-retroviral.

Los días de síntomas previos a su consulta, referidos por los pacientes, presentaban una importante variación de entre 1 y 120 días, siendo la mediana aproximada de 11 días.

Fiebre fue el síntoma referido con mayor frecuencia (77,7\% de los casos), seguido de alteración en el estado de conciencia $(72,2 \%)$ y cefalea $(58,3 \%)$. Signos meníngeos (rigidez de nuca), se encontró en sólo 54,5\% de los casos. En pacientes sin VIH versus con VIH/SIDA, los síntomas característicos como la fiebre, cefalea y rigidez de nuca se presentaron con mayor frecuencia $(85,7$ vs $72,7 \%)$, (78,6 vs, 45,4\%) y $(64,3$ vs 45,4\%), respectivamente, mientras que los pacientes con VIH/SIDA presentaban signos y síntomas más inespecíficos (90,9 vs 78,6\%). No hubo diferencias significativas entre ambos grupos con respecto al estado de conciencia alterado. 
En $86,1 \%$ de los casos, se presentaron otros síntomas, como son: 11 pacientes náuseas y vómitos $(30,5 \%)$, 5 fotofobia $(13,9 \%), 6$ astenia, adinamia y otros síntomas constitucionales $(16,6 \%), 10$ presentaron parálisis de un par craneal o foco motor $(27,8 \%)$ : III, VI y VII. Tres pacientes presentaron síndrome cerebeloso $(8,3 \%)$ y solo 2 pacientes presentaron convulsiones al ingreso $(5,5 \%)$.

Según la clasificación del Medical Research Council, se encontraban en el estadio II 19 pacientes $(52,7 \%)$ y un tercio eran Estadio III.

Diecinueve pacientes del total no presentaban infección extra-meníngea (52,7\% de los casos). Sí la presentaron los pacientes coinfectados por VIH/SIDA: del total de 22 pacientes, cinco tenían TBC pulmonar $(22,8 \%)$ y siete TBC miliar o diseminada (31,8\% de los casos).

\section{Análisis físico-químico del LCR}

Las características del LCR se resumen en la Tabla 2. El examen directo (tinción de Ziehl-Neelsen) no logró la observación de bacilos alcohol-ácido resistentes en paciente alguno.

Treinta y un pacientes tuvieron valores elevados de leucocitos, por arriba de $10 \times 10^{6} / \mathrm{mm}^{3}(86,1 \%)$, con predominio linfocitario con una mediana de $60 \%$. Se observó que los pacientes sin infección por VIH/SIDA presentaban mayor reacción inflamatoria en el LCR en comparación con los pacientes con VIH/SIDA, dado por el mayor valor de leucocitos (con una mediana de 240 vs 102,5 x 10\% $\mathrm{mm}^{3}$ ), proteínas (mediana de $188,5 \mathrm{vs} 107,5 \mathrm{mg} / \mathrm{dL}$ ) y ácido láctico (mediana de 6,15 vs 5,2 $\mathrm{mmol} / \mathrm{mL}$ ).

\section{Evaluación imagenológica}

Del total de 36 pacientes, a 22 pacientes $(61,1 \%)$ se les realizó sólo TC de encéfalo, a dos sólo RM (5,6\%) y a 12 pacientes $(33,3 \%)$ se les realizó TC y RM. Cabe remarcar que en algunos estudios presentaban más de un hallazgo patológico. De un total de 34 pacientes que se realizaron TC de encéfalo, 16 pacientes $(47,1 \%)$ no mostraban hallazgos patológicos significativos. En cambio, a los pacientes que se les realizó RM de cerebro, de un total de 16 pacientes, nueve $(56,2 \%)$ presentaban trastornos vasculares, siendo en dos de ellos trombosis pericallosa y en seis de ellos lesiones tipo vasculíticas difusas. Se desconoce, dado el tiempo que comprende el estudio, la razón para la escasa realización de RM en estos pacientes (Tabla 3).

Analizando diferencias entre pacientes con infección por VIH/SIDA y pacientes sin esta condición (Tabla 4), se observa que en los segundos es mayor el hallazgo de lesiones vasculares $(42,8$ vs $31,8 \%)$, mientras que en los con infección por VIH/SIDA se observan más cantidad de lesiones ocupantes de espacio tipo tuberculomas $(27,3$ vs 7,1\%), no apreciándose diferencias significativas en los otros posibles hallazgos entre ambos grupos.

Tabla 2. Características físico-químicas del LCR

\section{Total de pacientes}

$\begin{array}{ll}\text { Leucocitos x 10x6/mm³, mediana (rango) } & 116,5(0-900) \\ \text { Porcentaje de linfocitos en el LCR, mediana (rango) } & 60(20-80) \\ \text { Proteínas en LCR mg/dl, mediana (rango) } & 158,5(10->1.000) \\ \text { Glucosa en LCR mg/dl, mediana (rango) } & 30(3-77) \\ \text { Ac. Láctico en LCR mmol/mL, mediana (rango) } & 5,4(2-8,5)\end{array}$

\section{Total de pacientes VIH}

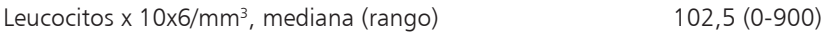

Porcentaje de linfocitos en el LCR, mediana (rango) 60 (20-80)

Proteínas en LCR mg/dl, mediana (rango) 107,5 (10-> 1.000)

Glucosa en LCR mg/dl, mediana (rango) 33,5 (5-77)

Ac. Láctico en LCR mmol/mL, mediana (rango) $\quad 5,2(1,6-8,1)$

\section{Total de pacientes no VIH}

Leucocitos $\times 10 \times 6 / \mathrm{mm}^{3}$, mediana (rango)

$240(14-500)$

Porcentaje de linfocitos en el LCR, mediana (rango)

$60(20-80)$

Proteínas en LCR mg/dl, mediana (rango)

$188,5(17-334)$

Glucosa en LCR mg/dl, mediana (rango)

$22(3-59)$

Ac. Láctico en LCR mmol/mL, mediana (rango)

$6,15(0-9,9)$

Tabla 3. Hallazgos patológicos en imágenes

\begin{tabular}{lcc} 
Hallazgo & $\begin{array}{c}\text { TC (\%) } \\
\mathbf{n = 3 4}\end{array}$ & $\begin{array}{c}\text { RM }(\%) \\
\mathbf{n}=16\end{array}$ \\
Refuerzo basal & $4(11,8)$ & $2(12,5)$ \\
Hidrocefalia & $9(26,5)$ & $3(18,75)$ \\
Alteraciones vasculares & $7(20,5)$ & $9(56,25)$ \\
Tuberculomas & $3(8,8)$ & $5(31,25)$ \\
Sin patologías & $16(47,1)$ & $1(6,25)$ \\
\hline
\end{tabular}

\begin{tabular}{lcc|}
$\begin{array}{l}\text { Tabla 4. Diferencias en imágenes entre pacientes no VIH } \\
\text { y VIH }\end{array}$ & $\begin{array}{c}\text { No VIH } \\
\mathbf{n}=\mathbf{1 4}\end{array}$ & $\mathbf{n}=\mathbf{2 2}$ \\
\hline Hallazgo & $2(14,3 \%)$ & $3(13,6 \%)$ \\
\hline Refuerzo basal & $4(28,6 \%)$ & $7(31,8 \%)$ \\
Hidrocefalia & $6(42,8 \%)$ & $7(31,8 \%)$ \\
\hline Alteraciones vasculares & $1(7,1 \%)$ & $6(27,3 \%)$ \\
\hline Tuberculomas & $5(35,7 \%)$ & $7(31,8 \%)$ \\
\hline Sin patologías & & \\
\hline
\end{tabular}




\begin{tabular}{|lrr|}
\hline \multicolumn{2}{|l|}{ Tabla 5. Resolución y secuelas en $\mathbf{3 6}$ casos de meningitis TBC } \\
\hline Inicio del tratamiento, mediana (rango), en días & 24 & $66,66 \%$ \\
\hline Tratamiento con corticosteroides al inicio (total, porcentaje) & 5 & $13,88 \%$ \\
Tratamiento quirúrgico (total, porcentaje) & 10 & $27,77 \%$ \\
Mortalidad (total, porcentaje) & 13 & $36,11 \%$ \\
\hline Secuela (total, porcentaje) & & \\
\hline
\end{tabular}

\section{Terapia anti TBC y co-adyuvante}

Veinticuatro pacientes recibieron tratamiento habitual (isoniazida, rifampicina, etambutol y pirazinamida) en un período de tiempo que comprendía desde el ingreso hasta 26 días después de haber ingresado (mediana de tiempo de inicio de 1 día) y 12 pacientes recibieron isoniacida, rifampicina, estreptomicina y pirazinamida. Del total, 24 pacientes $(66,6 \%)$ recibieron tratamiento co-adyuvante con dexametasona desde el ingreso.

Sobre tratamiento neuroquirúrgico, sólo cinco pacientes $(13,8 \%)$ requirieron neurocirugía: uno requirió ventriculostomía de urgencia y a los otros cuatro se le realizó derivación ventrículo-peritoneal.

\section{Evolución y resolución}

Trece pacientes $(36,1 \%)$ presentaron algún grado de secuela. Cabe aclarar que cinco pacientes $(13,8 \%)$ presentaban más de un trastorno secuelar al momento del alta (Tabla 5). Se registraron 10 muertes $(27,7 \%)$.

Secuelas al alta (del total): cuatro pacientes $(11,1 \%$ del total) presentaron algún grado de desorientación y bradipsiquia, ocho pacientes $(22,2 \%)$ presentaron un cuadro de tipo motor: uno de ellos presentó cuadriparesia, tres presentaron hemiplejía o hemiparesia grave izquierda, seis presentaron compromiso permanente de un par craneal (siendo los principales el III y el VI pares) y por último, un paciente $(2,7 \%)$ presentó como secuela síndrome de secreción inadecuada de hormona antidiurética (SIHAD) y otro $(2,7 \%)$ presentó hipertensión endocreaneal (HTE) permanente.

\section{Discusión}

Estudios recientes realizados en los países desarrollados han demostrado que la TBC meníngea es un problema vigente, que afecta en la mayoría de los casos a pacientes pediátricos y reciben tratamientos antituberculosos heterogéneos ${ }^{13,14}$. Nuestra casuística incluye 36 pacientes con diagnóstico definitivo de meningitis TBC, siendo diagnosticados y tratados todos en el Hospital Posadas de Buenos Aires, Argentina.

En esa casuística, la mediana de edad fue de 36,5 años, hallazgo que se correlaciona con una máxima expresión clínica descrita en la literatura médica entre los 35 y los 39 años, con una mayoría de sexo femenino.

En cuanto a las manifestaciones clínicas, más de tres cuartos de los pacientes presentaban fiebre al momento de la consulta, acompañado de otros síntomas como cefalea o alteración del estado de conciencia. La rigidez de nuca, signo meníngeo por excelencia, se presentó en aproximadamente la mitad de los pacientes, dato concordante con lo señalado en la bibliografía consultada. Una larga historia de enfermedad (más de 5-6 días) ha demostrado previamente ser una variable clínica altamente predictora de TBC meníngea ${ }^{15}$; en el presente trabajo se obtuvieron hallazgos similares a lo conocido, con una mediana de 11 días para todos los pacientes ${ }^{16,17}$.

Durante la hospitalización, la mitad de los casos fueron clasificados en Estadio II y un tercio eran Estadio III según la clasificación realizada por el Medical Research Council. Del total de pacientes, tres cuartas partes de los casos tenían alteración del estado de conciencia, sólo dos tenían convulsiones, y un tercio tenía paresia o plejía, todos los cuales fueron casos neurológicos graves como lo describe el estudio de Sütlas y cols. ${ }^{18}$. Sin embargo, la tasa de mortalidad en este estudio fue inferior al de otros informes a pesar de los casos eran graves y en etapa avanzada ${ }^{19}$.

En nuestra serie es destacable que un gran porcentaje de pacientes estaban co-infectados con VIH/SIDA, en concordancia con el alto riesgo de desarrollo de TBC en este grupo de pacientes. Se ha descrito un aumento de 20\% en el riesgo de contraer TBC en co-infección con VIH, en regiones en que la prevalencia de la TBC es alta ${ }^{20,21-}$.

En cuanto a la diseminación de TBC a otros parénquimas, más de la mitad de los pacientes no presentaba compromiso infeccioso en otros órganos, en comparación lo escrito en la literatura médica. El menor hallazgo de TBC extra-meníngea es un punto a analizar, pero dadas las características del trabajo, no creemos contar con información suficiente para poder dar una conclusión fehaciente ante dicho hallazgo, sino mencionar esta diferencia encontrada.

Un pilar fundamental que ayuda a sospechar el diagnóstico y a iniciar tratamiento anti-tuberculoso empírico es el análisis del LCR. La mayoría de los pacientes presentan alteraciones citoquímicas sugerentes de meningitis linfocitaria, con proteínas altas y glucosa baja, que se correlaciona con el diagnóstico posterior de meningitis tuberculosa. Estudios anteriores de Irán, India y Vietnam han encontrado que el porcentaje de linfocitos en el LCR es una de las variables de diagnóstico predictores más fuertes de TBC meníngea, especialmente cuando el recuento total es de menos de $1.000 \times 10^{322,23}$. Ninguno 
LCR, pero tiene como limitante no estar disponible en centros de menor complejidad y su alto costo, por lo que la sospecha clínica sigue siendo importante. Los estudios han demostrado que la precocidad en el inicio del tratamiento específico es determinante en la morbimortalidad de esta patología, por lo que es crucial tener elementos que orienten al diagnóstico precoz; son pilares fundamentales: las manifestaciones clínicas (antecedentes de enfermedad tuberculosa o clínica actual sugerente de TBC), antecedente de contacto epidemiológico, la radiografía de tórax y el estudio del LCR. Proponemos realizar en forma temprana una RM de encéfalo para la búsqueda de lesiones vasculares y granulomatosas, principalmente en pacientes con infección por VIH/SIDA.

Limitaciones a este análisis son su carácter retrospectivo, el extenso período de tiempo abarcado, que determinó disparidad en las decisiones clínicas y terapéuticas, cambios en la disponibilidad de recursos imagenológicos, la ausencia de la técnica de RPC para el diagnóstico etiológico, hoy herramienta indispensable para una confirmación diagnóstica precoz, cambios en los estándares de la terapia anti-retroviral en el SIDA y la necesidad de un mayor tiempo de seguimiento de los pacientes que superaron estas patologías. No se ha analizado cuánto fue la duración del tratamiento para comparar con las guías internacionales que sugieren seis meses, salvo en los casos graves, donde se aplican regímenes de tratamiento de 9-12 meses ${ }^{25}$.

Una alta proporción de los pacientes fueron tratados con dexametasona como adyuvante, componente bien establecido para el tratamiento de la TBC meníngea pues ha demostrado reducir significativamente las tasas de mortalidad. Diversos estudios han puesto en evidencia que el tratamiento anti-tuberculoso precoz y simultáneo con dexametasona mejora la sobrevida de pacientes con meningitis tuberculosa, sobre 14 años de edad, en forma independiente de la gravedad de la enfermedad ${ }^{26,27}$.

La letalidad fue una cifra inferior a la descrita con anterioridad en la literatura científica ${ }^{28}$. Podríamos atribuir esta baja letalidad al inicio precoz de la terapia específica $\mathrm{y}$ al uso de corticoterapia, pero no podemos dejar de tener en cuenta la muestra representada y la dificultad en el seguimiento a largo plazo.

La tasa de secuelas identificada en este trabajo fue elevada, similar a lo presentado en otros informes ${ }^{29}$.

Es de destacar que la meningitis tuberculosa continúa estando presente y ha experimentado un incremento en su incidencia en estas últimas décadas a consecuencia de la pandemia del VIH.

El diagnóstico de certeza precoz de esta patología es posible en la actualidad gracias a la técnica RPC en

\section{Conclusiones}

La meningitis tuberculosa continúa estando presente y ha experimentado un incremento en su incidencia a consecuencia de la pandemia del VIH. Su pronóstico depende de una precocidad en la sospecha diagnóstica, el buen uso de los recursos hospitalarios, haciendo hincapié en los estudios por imágenes y la instauración pronta de la terapia anti-tuberculosa, El rápido diagnóstico de certeza se logra a través de una nueva técnica de detección microbiológica: RPC de LCR, la que aún no se encuentra disponible en centros de mediana o baja complejidad, y resulta aún muy costosa, por lo que la sospecha clínica sigue siendo importante.

En cuanto al inicio de tratamiento, se debe optar, dentro de su disponibilidad, por fármacos de buena penetración al SNC, siempre en co-adyuvancia con corticosteroides, elaborando un esquema con al menos cuatro fármacos anti-tuberculosos, intensificado y prolongado.

Por último, aunque la TBC meníngea sólo afecte a un puñado de personas cada año, el médico debe estar preparado para tratarla empíricamente si ha surgido la sospecha de dicha patología, y esperar a descartarla en un tiempo posterior. 


\section{Referencias bibliográficas}

1.- Bozluolcay M, Pelin Z. Tuberculosis of the central nervous system in Turkey: a retrospective study of 90 adult patients. J Neurol Sci, 2003; 20 (2): 120-6. https://doi.org/10.1100/2012/169028.

2.- Brancusi F, Farrar J, Heemskerk D. Tuberculous meningitis in adults: a review of a decade of developments focusing on prognostic factors for outcome. Future Microbiol 2012; 7: 1101-6. http://dx.doi.org/10 .2217/fmb.12.86

3.- Girardi E, Raviglione M C, Antonucci G, Godfrey-Faussett P, Ippolito G. Impact of the HIV epidemic on the spread of other diseases: the case of tuberculosis. AIDS 2000; 14 (Suppl 3): 47-56. PMID: 11086849.

4.- Vinnard C, Macgregor R. Tuberculosis meningitis in HIV infected individuals. Curr HIV/AIDS Rep 2009; 6: 139-45. https://doi. org/10.1007/s11904-009-0019-7.

5.- Thwaites G, Fisher M, Hemingway C, Scott G, Solomon T, Innes J. British Infection Society guidelines for the diagnosis and treatment of tuberculosis of the central nervous system in adults and children. J Infect 2009; 59 (3): 167 87. doi: 10.1016/j.jinf.2009.06.011.

6.- Marais S, Thwaites G, Schoeman J F, Török M E, Misra U K, Prasad K, et al. Tuberculous meningitis: a uniform case definition for use in clinical research. Lancet Infect Dis 2010; 10 (11): 803-12. https://doi.org/10.1016/s14733099(10)70138-9.

7.- Thwaites G E, Tran T H. Tuberculous meningitis: many questions, too few answers. Lancet Neurol 2005; 4(3): 160e70. doi 10.1016/S1474-4422(05)01013-6.

8.- Marais S, Pepper D, Schutz C, Wilkinson R J, Meintjes G. Presentation and outcome of tuberculous meningitis in a high HIV prevalence setting. PloS One. 2011; May (6). Issue 5.e2077. doi: 10.1371/journal. pone. 0020077 .

9.- Abdelmalek R, Kanoun F, Kilani B, Tiouiri H, Zouiten F, Ghoubantini A, et al. Tuberculous meningitis in adults: MRI contribution to the diagnosis in 29 patients. Int $\mathrm{J}$ Infect Dis 2006; 10(5): 372e7. doi: 10.1016/j. ijid.2005.07.009

10.- Oztoprak I, Gumus C, Oztoprak B, Engin
A. Contrast medium enhanced MRI findings and changes over time in Stage I tuberculous meningitis. Clin Radiol 2007; 62 (12): 1206e15. doi: 10.1016/j.crad.2007.06.001.

11.- van Loenhout-Rooyackers J H, Keyser A, Laheij R J, Verbeek A L, van der Meer J W. Tuberculous meningitis: is a 6-month treatment regimen sufficient? Int J Tuberc Lung Dis 2001; 5(11): 1028e35. PMID: 11716339.

12.- Prasad K, Singh M B. Corticosteroids for managing tuberculous meningitis. Cochrane Database Syst Rev 2008; (1): CD002244. doi: 10.1002/14651858.CD002244.pub3.

13.- García-Martos P, Castaño M A, Canueto J, Hohr L, González-Borrachero M L. Tuberculosis en 34 pacientes con infección por VIH. Enferm Infec Microbiol Clin 1991: 64-6. PMID: 1805951.

14.- de Souza C H, Yamane A, Pandini J C, Ceretta L B, Ferraz F, da Luz G D, et al. Incidence of tuberculous meningitis in the State of Santa Catarina, Brazil. Rev Soc Bras Med Trop. 2014; 47: 483-9. doi: 10.1590/00378682-0122-2014

15.- Kumar R, Singh S N, Kohli N. A diagnostic rule for tuberculous meningitis. Arch Dis Child 1999, 81(3): 221-4. doi: 10.1136/adc.81.3.221.

16.- Schaller M A, Wicke F, Foerch C, Weidauer S. Central nervous system tuberculosis: etiology, clinical manifestations and neuroradiological features. Clin Neuroradiol. 2019; 29(1): 3-18. doi: 10.1007/s00062-018-0726-9.

17.- Enberg M, Quezada M, de Toro C, Fuenzalida L. Meningitis tuberculosa en adultos: análisis de 53 casos. Rev Chilena Infectol 2006; 23 (2): 134-9. doi: 10.4067/s071610182006000200006.

18.- Sütlas P N, Unal A, Forta H, Senol S, Kirbas D. Tuberculous meningitis in adults: review of 61 cases. Infection 2003; 31 (6): 387-91, 2003. doi: 10.1007/s15010-003-3179-1.

19.- Hosoglu S, Geyik M F, Balik I, Aygen B, Erol $\mathrm{S}$, Aygencel TG, et al. Predictors of outcome in patients with tuberculous meningitis. Int J Tuberculosis Lung Dis 2002; 6 (1): 64-70. PMID: 11931403.

20.- Zumla A, Malon P, Henderson J, Grange J. Impact of HIV infection on tuberculosis. Postgrad Med 2000; 76: 259-68. doi: 10.1136/ pmj.76.895.259.

21.- Dube M, Holtom P, Larsen R. Tuberculous meningitis in patients with and without human immunodeficiency virus infection. Amer J Med 1992; 93: 520-4. doi: 10.1016/00029343(92)90579-z.

22.- Marais S, Pepper D J, Schutz C, Wilkinson R J, Meintjes G. Presentation and outcome of tuberculous meningitis in a high HIV prevalence setting. PloS one. 2011; 6(5): e20077. doi: 10.1371/journal.pone.0020077.

23.- Moghtaderi A, Alavi-Naini R, Izadi S, Cuevas L E. Diagnostic risk factors to differentiate tuberculous and acute bacterial meningitis. Scand J Infect Dis 2009; 41(3): 188-94. doi: 10.1080/00365540902721384.

24.- Youssef F G, Afifi S A, Azab A M, Wasfy M M, Abdel-Aziz K M, Parker T M, et al. Differentiation of tuberculous meningitis from acute bacterial meningitis using simple clinical and laboratory parameters. Diagn Microbiol Infect Dis 2006; 55(4): 275-8. doi: 10.1016/j. diagmicrobio.2006.01.027.

25.- Pienaar M, Andronikou S, van Toorn R. MRI to demonstrate diagnostic features and complications of TBM not seen on CT. Childs Nerv Syst 2009; 25: 941-7. doi: 10.1007/ s00381-008-0785-3.

26.- Cresswell F V, Te Brake L, Atherton R, Ruslam R, Dooley K E, Aarnoutse R, et al. Intensified antibiotic treatment of tuberculosis meningitis Exp Rev Clin Pharmacol 2019; 12(3): 267-88. doi: 10.1080/17512433.2019.1552831

27.- Dooley D, Carpenter J, Rademager S. Adjuntive corticosteroid therapy for tuberculosis: A critical reappraisal of the literature. Clin Infect Dis 1997; 25: 872-87. doi: 10.1086/515543.

28.- Thwaites G E, Nguyen D B, Nguyen H D, Quy H T, Tuong Oanh D T, Cam Thoa N T, et al. Dexamethasone for the treatment of tuberculous meningitis in adolescents and adults. N Engl J Med 2004; 351: 1741-51. doi: 10.1056/NEJMoa040573.

29.- Verdon R, Chevret S, Laissy J P, Wolff M. Tuberculous meningitis in adults: Review of 48 cases. Clin Infect Dis 1996; 22: 982-8. doi: 10.1093/clinids/22.6.982.

30.- Merkler A E, Reynolds A S, Gialdini G, Morris N A, Murthy S B, Thakur K, et al. Neurological complications after tuberculous meningitis in a multi-state cohort in the United States. J Neurol Sci 2017; 375: 460-3. doi: 10.1016/j. jns.2017.02.051. 